\title{
The bottom line for lesion localization in breast cancer
}

\author{
M. Ahmed ${ }^{1}$
}

Received: 21 April 2020 / Accepted: 8 June 2020 / Published online: 13 June 2020

(c) Springer Science+Business Media, LLC, part of Springer Nature 2020

\begin{abstract}
The development of radioisotope-independent, wire-free localization techniques for excision of non-palpable breast cancer will continue to expand rapidly within the next few years, increasing market competition. It is imperative that the introduction of these new technologies is correctly evaluated in terms of their major logistical benefits in streamlining pathways from radiology to the operating theatre and subsequent financial savings, rather than focusing upon margin re-excision rates-in which they are unlikely to advance current practice.
\end{abstract}

The last five years have seen a rapid expansion in the number of techniques offering an alternative to the historical standard of wire-guided localization (WGL). The only options previously available had been radioisotope dependent localization techniques-which never gained mainstream acceptance due to handling, disposal and accessibility issues associated with radiation - and intraoperative ultrasound - which remains a niche of surgical enthusiasts trained in ultrasound. However, now there are three-radioisotopefree-licensed localization devices for use in Europe and the USA, comprising the magnetic technique using Magseed (Endomag, Cambridge, UK), radiofrequency detection using the Localizer (Hologic, Mass, USA) and infrared detection with the Savi Scout (Cianna Medical, Aliso Viejo, USA). In addition to these there are additional magnetic localization techniques in development and nearing market availability from the Takumi magnetic probe (Matrix Cell Research, Tokyo, Japan). MaMaLoc (Sirius Medical, Eindhoven, The Netherlands) and MOLLI (Sunnybrook Health Sciences, Toronto, Canada) systems.

With this recent rapid proliferation of technology, the decision to shift to new localization techniques or not and if so, which type can be a difficult decision to make for clinicians and healthcare providers. The three current main players of Endomag, Hologic and Cianna Medical have all published several cohort series demonstrating

M. Ahmed

muneer.ahmed@ucl.ac.uk

1 Division of Surgery and Interventional Science, University College London, Royal Free Hospital, 9th Floor (East), Pond Street, London NW3 2QG, UK the feasibility of their products to perform safe localized lesion excisions and this is an essential first step in the utilization of any such technology. Interestingly, these companies have not embraced the results from the work, which was performed on radioguided localization surgery (RGL) - comprising radioactive seed localization (RSL) and radioguided occult lesion localization (ROLL) - the principle on which all these 'new' technologies are based. They are a replication of radioactive seed localization, without the radioactivity. The results of all the work comparing RGL with WGL - including meta-analysis-never managed to identify a significant difference in re-excision rates between the 2 techniques [1]. One of the most robust randomized controlled trials performed by Postma et al [2] powered the difference in the two arms at 15 per cent, with the final figure in the trial only being 2 per cent - underpowering the trial. Yet, all the cohort series - plus or minus a retrospective control arm - have focused upon re-excision rates as their main outcome measure after successful localization for their studies. Unsurprisingly, there has been no significant difference identified in any of their results-over approximately 25 studies to date. This underlies the lack of focus upon answering the key issues, which require addressing when considering localization-guided breast surgery. Only one trial by Srour et al. [3] touched on it and they identified in their retrospective cohort-controlled group study comparing WGL, RGL and SAVI Scout, that the preoperative time and delays to the operating theatre were significantly reduced by application of wire-free technologies. This finally starts to address the area where wire-free technologies have their greatest potential role. Within the UK many NHS Trusts have 
undergone mergers, resulting in multiple hospital working sites. The flexibility of wireless localization technologies allows utilization of radiological capacity at multiple sites prior to the day of surgery-even if all surgery is to be performed at a single site. This streamlines the patient journey and most importantly allows them to avoid extra procedures-at a stressful time-or overburdening busy radiology units at the operating site on the morning of surgery. The importance of this cannot be underestimated as many hospitals even within the same trust use different online data systems and for radiologists to access precise diagnostic details for complex localizations from different sites can be very difficult, time consuming and prone to error [4]. This then leads to the key determinant factor of these technologies, which is facilitating the commencement of surgery on time and maximization of theatre capacity for cancer work. By having a cancer patient 'ready to go' first on the list in the morning-with their localization complete - avoids the necessity to either delay the start of the operating list or place a small, benign case to fill the shortfall in time before the wire-localization is complete. With the cost of NHS operating theatre time priced at a premium of between $£ 20-60$ per minute, [5] this can mean a significant cost saving and also assistance in preventing national cancer treatment target breaches through maximization of cancer cases operated upon.

The next and bottom line in uptake of most techniques within any healthcare system is of course cost. The wire is very cheap in comparison to these technologies, with each wireless marker being at least five times its price. Therefore, at face value these new technologies have hit a stumbling block, factor in the initial setup costs with the handheld console units and the situation looks bleak. Of course, this is not necessarily the case, when one integrates streamlining of the patient pathway from radiology to surgery, but this needs to be accurately assessed. There is no published data to determine the economic benefits of radioisotope-independent, wire-free technologies. This is absolutely imperative in order to justify the utilization of these technologies. As alluded to earlier, the current three main players in this field will not remain alone for long. With the incoming explosion of these technologies on the horizon, RCTs using re-excision rates as their primary outcome measures will be unproductive. It is clear that these technologies provide safe localization comparable to the wire, but also it must be emphasized, that they are not superior to the wire in this regard. These technologies possess the potential benefit of streamlining management, but this must be formally demonstrated together with their economic viability to achieve this. I would therefore urge the current and future entrepreneurs creating rapid developments in this exciting field to engage constructively in trials, which not only focus upon the feasibility of localization but also seriously produce objective metrics for the core principles of logistic and cost-benefits, which up until now have been neglected from this expanding field of breast cancer management.

Ultimately, lesion localization surgical innovations would benefit from structured evaluation to facilitate their implementation. Such structured evaluation would accelerate utilization - for evaluation - in individual institutions and reduce device-related complications [6]. It would also allow formal evaluation - beyond individual institutions - to allow integration of such technologies into widespread clinical practice. Currently, within the context of the 5-stage IDEAL Framework [7], these technologies have not been assessed beyond a development stage (2A). They need to progress into the exploration stage (2B) of prospective, multicentre evaluation and pilot/feasibility RCTs. Pending these findings, the assessment stage in terms of RCTs (3), including alternative designs before the long-term monitoring phase using registries (4). This is reinforced by the lack of current progression along the 'diffusion cascade' of surgical innovation beyond an idea-conversion (clinical) to implementation stage (RCTs) [8]. These shortcomings in evidence must be addressed in order to rigorously evaluate these devices.

Ultimately, which device - if any or none at all of the multitude that will be available-a unit selects will come down to personal preferences of clinicians in view of the nuanced differences between devices. However, the bottom line of the economic justification of whichever technology (old or new) is selected or retained has to be transparently in place and formally confirmed through the structured processes used to evaluate surgical innovations.

\section{Compliance with Ethical Standards}

Conflict of interest MA has received an unrestricted educational grant from Endomag previously. MA has received honorarium from Hologic BVBA. This article does not contain any studies with human participants or animals performed by the author.

\section{References}

1. Ahmed M, van Hemelrijck M, Douek M (2013) Systematic review of radioguided versus wire-guided localization in the treatment of non-palpable breast cancers. Breast Cancer Res Treat 140(2):241-252

2. Postma EL, Verkooijen HM, van Esser S, Hobbelink MG, van der Schelling GP, Koelemij R, Witkamp AJ, Contant C, van Diest PJ, Willems SM, Borel Rinkes IH, van den Bosch MA, Mali WP, van Hillegersberg R (2012) (2012) Group Rs Efficacy of 'radioguided occult lesion localisation' (ROLL) versus 'wire-guided localisation' (WGL) in breast conserving surgery for non-palpable breast cancer: a randomised controlled multicentre trial. Breast Cancer Res Treat 136(2):469-478 
3. Srour MK, Kim S, Amersi F, Giuliano AE, Chung A (2020) Comparison of wire localization, radioactive seed, and Savi $\operatorname{scout}((\mathrm{R}))$ radar for management of surgical breast disease. Breast J 26(3):406-413

4. Ahmed M, Wood S, Leff D (2019) An evaluation of access to critical breast oncoplastic patient information within a regional autologous free-flap reconstruction service. Ecancermedicalscience 13:92

5. Scotland PH. Data and intelligence - Theatres. 2019.

6. Marcus RK, Lillemoe HA, Caudle AS, Weinberg JS, Gidley PW, Skibber JM, Levenback CF, Swisher SG, Aloia TA (2019) Facilitation of surgical innovation: is it possible to speed the introduction of new technology while simultaneously improving patient safety? Ann Surg 270(6):937-941

7. Hirst A, Philippou Y, Blazeby J, Campbell B, Campbell M, Feinberg J, Rovers M, Blencowe N, Pennell C, Quinn T, Rogers W,
Cook J, Kolias AG, Agha R, Dahm P, Sedrakyan A, McCulloch P (2019) No surgical innovation without evaluation: evolution and further development of the IDEAL framework and recommendations. Ann Surg 269(2):211-220

8. Garas G, Cingolani I, Panzarasa P, Darzi A, Athanasiou T (2017) Network analysis of surgical innovation: measuring value and the virality of diffusion in robotic surgery. PLoS ONE 12(8):e0183332

Publisher's Note Springer Nature remains neutral with regard to jurisdictional claims in published maps and institutional affiliations. 